\title{
A PRECISION METHOD OF CALIBRATING A TUNING FORK BY COMPARISON WITH A PENDULUM
}

\author{
By Charles Moon
}

ABSTRACYT

A photographic method for determining the relative frequency of a tuning fork and pendulum is described in which no energy is drawn either from the fork, the pendulum, or from the fork-driving circuits. A photographic record is obtained from which the frequency of the fork, the amplitude of the fork, and the amplitude of the pendulum are obtained. The accuracy of the timing is such that the time interval for an integral number of fork vibrations can be determined to 20 microseconds. If the pendulum is timed over an interval of one second, the error in frequency will be \pm 0.002 per cent. If the time interval is increased to $n$ seconds, the error will be $\pm \frac{1}{n} \times 0.002$ per cent.

Irregularities in relative frequency have been found which are thought to be due to a variable rate of the pendulum caused by microseismic movements of the building. Curves are given showing the frequency amplitude relation for two forks (one of steel and one of elinvar) when vibrating freely.

\section{CONTENTS}

II. Description of method and

III. The amplitude of the fork

IV. Frequency of the fork

V. Observational errors in $P$ and $D$

VI. Accuracy attainable....... 216

\section{INTRODUCTION}

A summary of methods for calibrating tuning forks has been given recently by Klein and Rouse. ${ }^{1}$ Since the publication of this summary a method using some ideas taken from the work of Rayleigh ${ }^{2}$ and some from the work of $\mathrm{Reed}^{3}$ has been described by Curtis and Moon. ${ }^{4}$ The arrangement is such that the fork is rated against a freely vibrating pendulum by an optical method which does not draw any energy from either the fork or the pendulum. A refinement of this method adapted for photographic recording is described in this paper. The results obtained have apparently about the ultimate accuracy possible by this or, perhaps, by any other method, since there is evidence of lack of perfect isochronism in either the fork or the pendulum.

\section{DESCRIPTION OF METHOD AND APPARATUS}

Three records are traced simultaneously on a moving film. The amplitude of the fork is obtained from one of these records, the frequency comparison and the amplitude of the pendulum from

1 J. Opt. Soc. Am. and Rev. Sci. Inst., 14, p. 263; 1927.

2 Nature, 17, p. $12 ; 1877$.

'Phys. Rev., 12, p. 282; 1901.

1 Absolute Measurement of Capacitance by Marwell's Method, B. S. Sci. Paper No. 564, B. S. Bul., 22, p. 487 . 
the other two. The only other data needed are a few fixed distances. A sketch showing the general arrangement of the apparatus is given in Figure 1.

Light from the arc lamp, $X$, is focused on the slit, $S_{1}$, of the tuning fork shutter by the condensing lens, $L_{1}$. An image of $S_{1}$ is formed on the film by each of the concave mirrors $M$ and $N$; $M$ being fastened to the pendulum and $N$ to the pendulum housing. When the pendulum is at rest, these images coincide. When the pendulum and fork are both vibrating, the images from $M$ are separated by the rotary motion of the mirror and a single dash is recorded on the film for each opening of the shutter. The distance between the dashes on the film is proportional to the amplitude of the pendulum. The speed of the film is such that on the return swing of the pendulum the dashes are distinct from those on the previous swing. These dashes will be hereafter called "timing lines." The images from the mirror $N$ overlap so as to trace a solid line on the film, which is at the center of the path of the images formed by $M$. Some

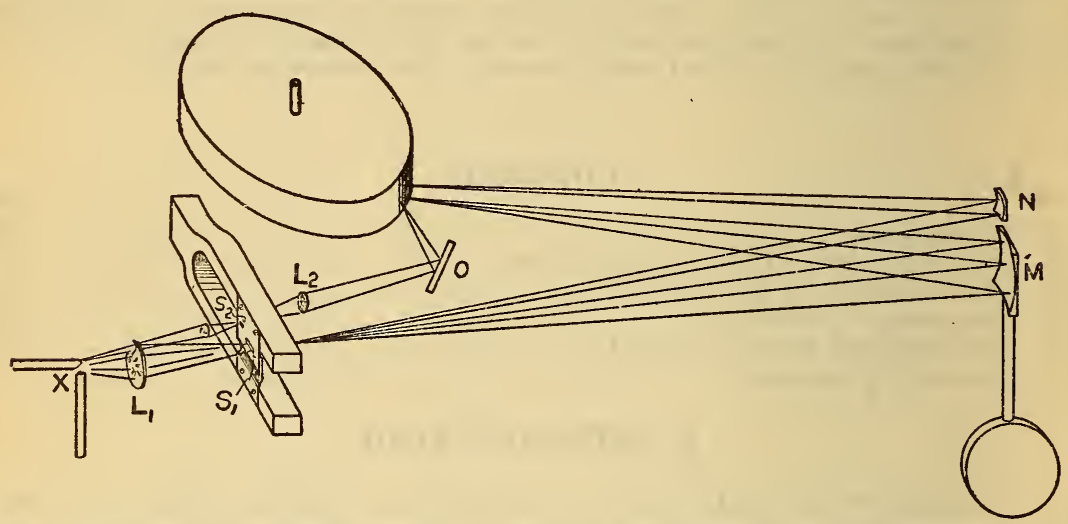

FigURE 1.-Schematic diagram showing arrangement of apparatus

light is also focused on the slit, $S_{2}$, cut in one vane of the shutter at a place not overlapped by the other vane. The image of $S_{2}$ formed on the film by the lens, $L_{2}$, and the mirror, $O$, is a broad band from which the amplitude of the fork may be determined.

Parts of film records are shown in Figure 2; $(a)$ was made with the fork driven at constant amplitude; $(b)$ with the fork vibrating freely.

\section{THE AMPLITUDE OF THE FORK}

The amplitude of the fork is obtained from the width of the broad band which in the widened image of the slit $S_{2}$ formed by the lens $L_{2}$ and the mirror $O$. The width of this band is proportional to $K X+C$, where $X$ is the actual double amplitude of the fork; $K$ a constant depending on the magnification of the lens $L_{2}$, and $C$ the width of the band at zero amplitude. The constants $K$ and $C$ can best be obtained by experiment. 


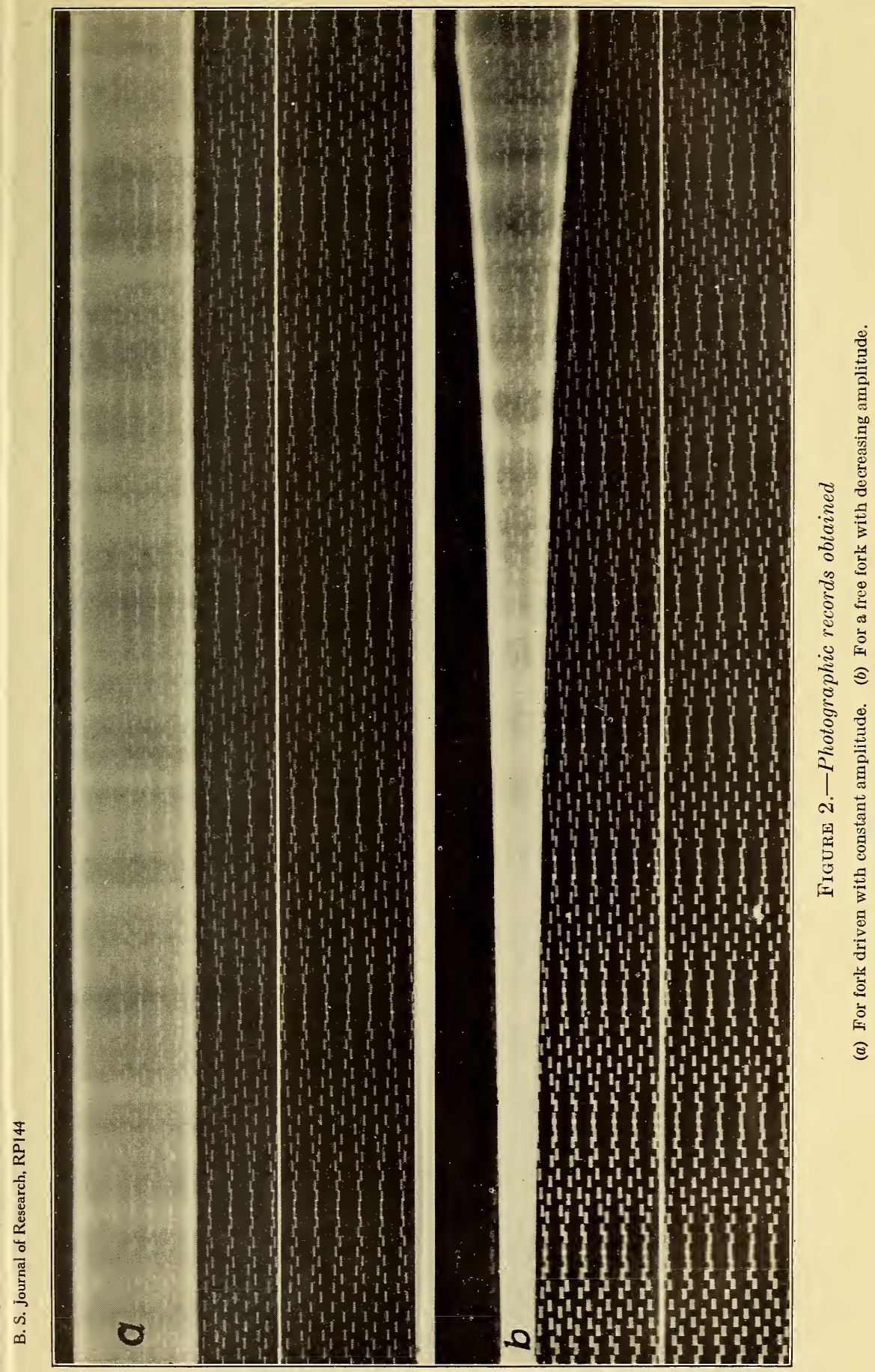




\section{FREQUENCY OF THE FORK}

If the film were wide enough to record the complete swing of the pendulum, the images formed by the mirror $M$ would fall on a sine curve. Actually only a small part of the sine curve on each side of the axis is recorded so that the film shows for each pendulum swing only a few timing lines forming a column, which over the central part of the path is nearly at right angles to the axis of the curve. The images formed by the mirror $N$ trace the axis of the sine curve on the film.

If nearly an exact integral relation exists between the frequency of the fork and the pendulum, there will be a small uniform progression of the timing lines recorded on successive swings, so that the timing lines are also arranged in two series of diagonal rows, one series when the pendulum is swinging forward and one when it is swinging backward.

These series intersect the axis at regular intervals.

To compute the frequency of the fork, let

$P=$ progression of the timing line in one complete vibration of the pendulum,

$D=$ distance between timing lines one-half fork cycle apart measured at the center of the path of image formed by mirror $M$, Figure 1,

$N=$ frequency of pendulum in cycles per second,

$F=$ frequency of fork, nominal value known,

$R=$ nominal ratio of the frequency of fork to the frequency of

Then the pendulum,

$D=$ number of pendulum vibrations for the fork to gain or lose $\bar{P}$ one-half fork vibration on the pendulum.

or

$2 D=$ number of seconds for the fork to gain one vibration on the $\overline{N P}$ pendulum,

the reciprocal of this quantity or $\frac{N P}{2 D}$ is the number of cycles per second that the fork gains or loses on the pendulum, so that

$$
F=N R \pm \frac{N P}{2 D}
$$

The sign of $N P / 2 D$ will be positive if the fork is gaining on the pendulum and negative if the pendulum is gaining on the fork.

If the pendulum is gaining on the fork the progression will be up on the forward swing of the pendulum and the rows of dashes recorded will have a positive angle with the axis. The dashes recorded on the backward swings will have a negative angle.

If the fork is gaining on the pendulum the progression will be opposite to that given in each case.

Assuming that $N$ is known with sufficient precision, the error in $F$ computed from formula (1) is largely due to the error in $P$ because $D$ is usually from 10 to 40 times as large and can be measured with all the accuracy required. Furthermore approximate coincidences of the timing lines with the axis will occur every several swings of the pen- 
dulum so that $P$ can be expressed in terms of $D$. If $n$ is the number of coincidences in the total time $t$ then

$$
P=\frac{n D \pm \Delta}{t}
$$

where $\Delta$ is a small quantity which shows how much the total progression lacks of making exactly $n$ coincidences. The sign of $\Delta$ will be positive if the total progression is slightly more than enough to make $n$ coincidences and negative if slightly less than enough.

\section{OBSERVATIONAL ERRORS IN P AND D}

The errors in measuring $P$ and $D$ are due to a number of causes:

(a) Timing lines which are sharp and well defined for large amplitudes of the fork become widened and diffuse for small amplitudes. With a free running fork the position of timing lines can be determined with an error of $0.02 \mathrm{~mm}$ when the double amplitude of the fork is 4 $\mathrm{mm}$, but when the double amplitude falls to $0.5 \mathrm{~mm}$ the measured position may be in error by $0.1 \mathrm{~mm}$.

(b) If the adjustment of the shutter vanes is such that the openings do not coincide in the rest position, the timing lines will not be evenly spaced, so that care must be taken to get an average value of $D$. For the same reason, on a free fork record, measurements of $P$ must be taken on two adjacent rows of lines and then averaged. A complete discussion of the behavior and adjustment of the tuning fork shutter is given by Curtis and Duncan. ${ }^{5}$

(c) The image formed by the mirror $M$ sweeps across the film with a motion which is nearly simple harmonic. Hence, the distance between timing lines decreases as the distance from the axis increases, and the motion can be considered uniform only near the axis or center of the path. If measurements to timing lines at a distance from the axis are necessary, a correction must be made which will depend on the actual apparatus used. This correction can be easily computed from the equations of simple harmonic motion.

\section{ACCURACY ATTAINABLE}

The discussion of accuracy attainable will be limited to the particular apparatus that has been used. The pendulum is of invar with chromium-plated steel knife-edge supported on a sapphire plane; the nominal frequency was two cycles per second. A vacuum of approximately $0.1 \mathrm{~mm}$ of mercury was maintained in the pendulum housing. Two excellent 100-cycle forks, one of high-carbon steel and another of elinvar were used. Both forks were very heavy with prongs $35 \mathrm{~cm}$ long. The pendulum, forks, and camera were mounted on brackets bolted to the walls on the second story of a brick building.

The accuracy of the relative frequency measurements, assuming that the pendulum and the fork execute perfect vibrations, depends on the time interval of the observation. When the pendulum is vibrating with a semiarc of $0.5^{\circ}$, the distance between timing lines recorded 0.01 second apart is about $10 \mathrm{~mm}$, and since the position of

- A ccurate Measurements of Short Time Intervals, B. S. Sci. Paper No. 470, B. S. Bul., 19, p. 17; 1923. 
a timing line can be measured to $\pm .02 \mathrm{~mm}$, the time can be measured to $\frac{0.02}{10} \times 0.01$, or 20 microseconds.

If the fork is rated over a time interval of one complete vibration of the pendulum or 0.5 second, the error in frequency will be 40 parts in $1,000,000$. By increasing the time of observation sufficiently any desired accuracy can be obtained. If the time interval is increased to five seconds, the error will be 4 parts in 1,000,000.

The curve in Figure 3 shows two independent sets of measurements on the same film by two observers. The variation between the two sets is at no place greater than 0.0005 per cent. The frequencies plotted in this curve were determined for 5 -second intervals.

There is some doubt as to how far the determination of relative frequencies may be safely carried, because of the uncertainty in the pre-

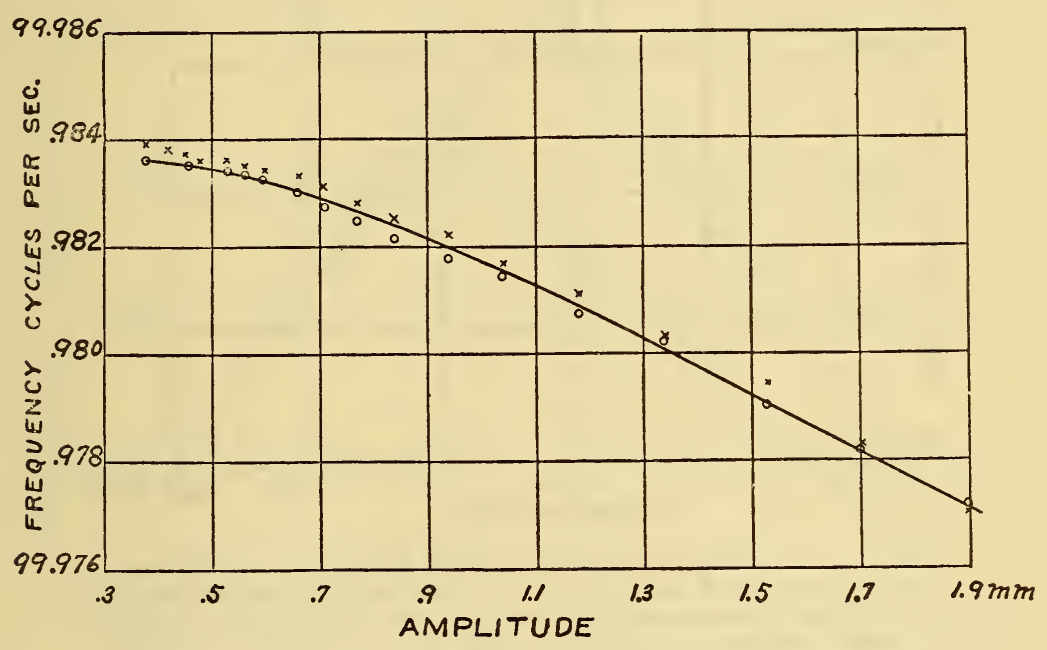

FIGURE 3.-Variation in results obtained by different observers on the same film record

cision of individual vibrations of the fork and pendulum. That a good pendulum can maintain an average frequency over a long interval of time with great precision is definitely established, but at the same time there may be a variation in single vibrations or in a group of a few vibrations due to microseismic movements of the pendulum support.

Figure 4 shows two frequency curves of the steel fork by comparison with the pendulum from records taken on two calm nights. The broken curve is typical of the results usually obtained. The solid curve has periodic fluctuations occuring at intervals of about 10 seconds. These fluctuations are too large to be attributed to experimental error, and are believed to be due to variations in the pendulum rate caused by vibration of the building. As a rule, when fluctuations have been observed they have been irregular and not periodic 
as shown in Figure 5. A disturbance of short pendulums by earth tremors has been reported by G. R. Putnam, ${ }^{6}$ who says:

A curious phenomenon was noted in connection with the use of these short pendulums. When lowered on the knife-edge and brought perfectly to rest, they were found in a short time to take up a slight oscillation, amounting sometimes to two minutes of arc. This was undoubtedly the effect of earth tremors, and was noted at all places where they were used, including the summit of Pikes Peak.

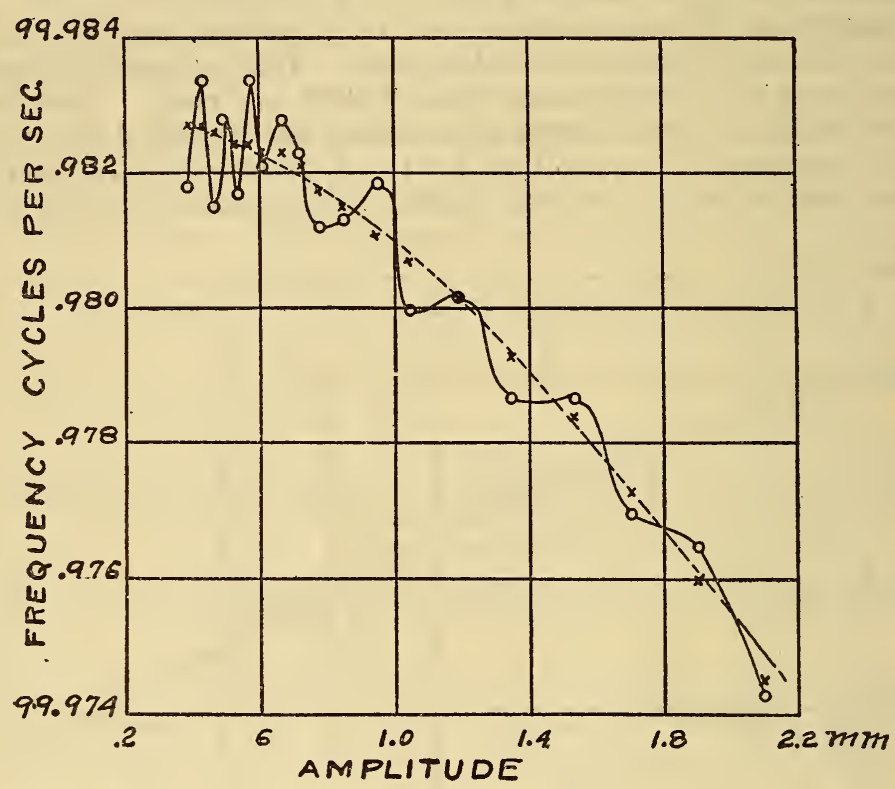

FIGURE 4.-Solid curve shows apparent fluctuations in frequency of a fork which are thought to be actual fluctuations in the pendulum rate caused by earth tremors. Dotted curve shows results obtained at another time with the same fork and pendulum

Mr. Putnam believed that the earth tremors caused no uncertainty in the period of his pendulums, but his pendulums were rated over intervals of several hours.

The two curves in Figure 5 show the change of frequency with amplitude of the two forks when the driving power was cut off and the amplitude allowed to decrease. The driving coils and laminated

\footnotetext{
${ }^{6}$ Report of the Superintendent of the U. S. Coast and Geodetic Survey for 1894, pp. 39-40, pt. 2.
} 
pole pieces, which were of the type described by Eckhardt, Karcher, and Keiser, ${ }^{7}$ were left in place. No permanent magnets are used in this drive.

A number of interesting problems have been suggested but are without the scope of this work, which has been carried out as a necessary side problem in some absolute electrical measurements.

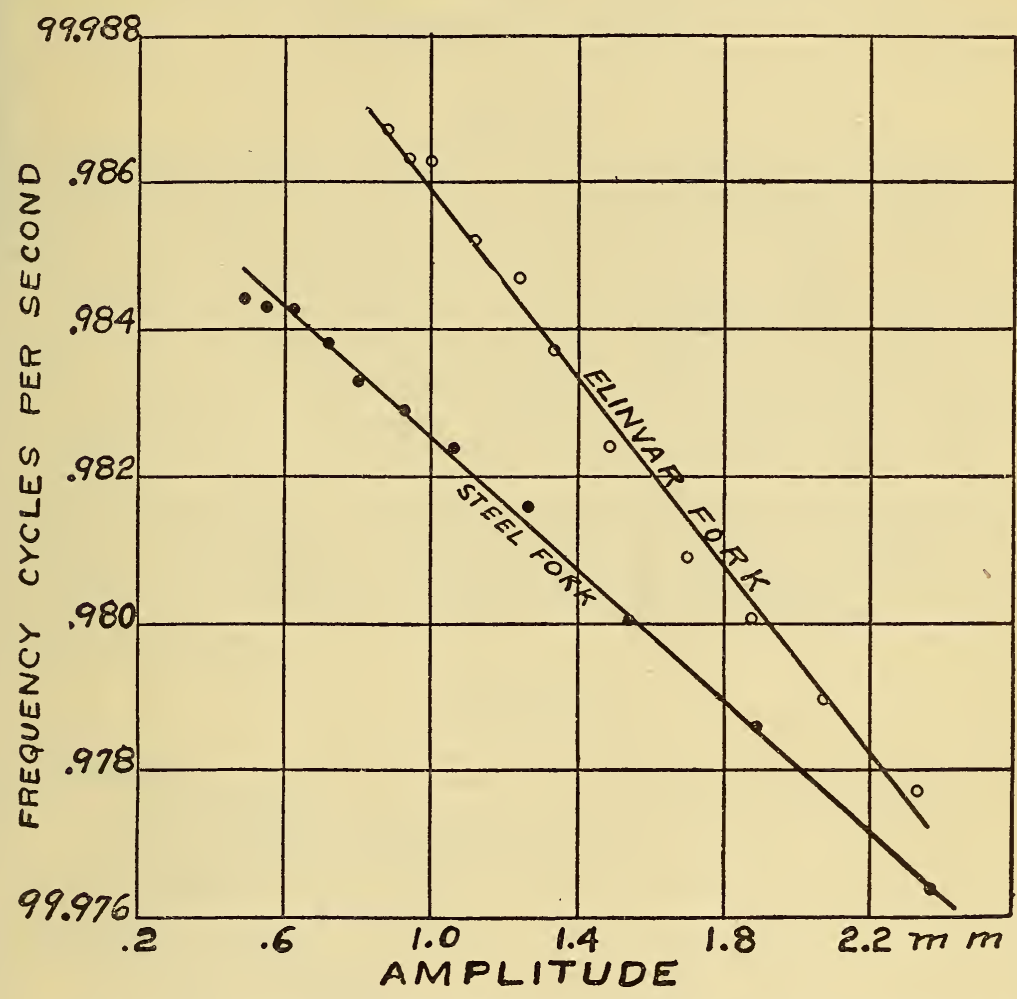

FIGURE 5.-Variation of frequency with amplitude for two 100-cycle forks

The writer is indebted to Dr. H. L. Curtis for suggestions and helpful criticism.

Washington, June 15, 1929.

7 Phys. Rev., 17, p. 535, 1921. 